ARTICLE

\title{
Control of electronic band profiles through depletion layer engineering in core-shell nanocrystals
}

Michele Ghini ${ }^{1,2,3,7}$, Nicola Curreli (10) ${ }^{1,7 凶}$, Matteo B. Lodi ${ }^{4}$, Nicolò Petrini ${ }^{1,5}$, Mengjiao Wang ${ }^{1,6}$, Mirko Prato (i) ${ }^{6}$,

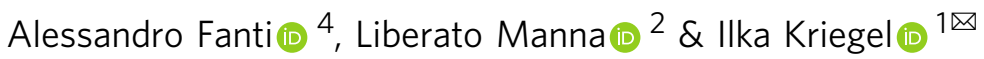

Fermi level pinning in doped metal oxide (MO) nanocrystals (NCs) results in the formation of depletion layers, which affect their optical and electronic properties, and ultimately their application in smart optoelectronics, photocatalysis, or energy storage. For a precise control over functionality, it is important to understand and control their electronic bands at the nanoscale. Here, we show that depletion layer engineering allows designing the energetic band profiles and predicting the optoelectronic properties of MO NCs. This is achieved by shell thickness tuning of core-shell $\mathrm{Sn}: \mathrm{In}_{2} \mathrm{O}_{3}-\mathrm{In}_{2} \mathrm{O}_{3} \mathrm{NCs}$, resulting in multiple band bending and multi-modal plasmonic response. We identify the modification of the band profiles after the light-induced accumulation of extra electrons as the main mechanism of photodoping and enhance the charge storage capability up to hundreds of electrons per NC through depletion layer engineering. Our experimental results are supported by theoretical models and are transferable to other core-multishell systems as well.

\footnotetext{
${ }^{1}$ Functional Nanosystems, Istituto Italiano di Tecnologia, via Morego 30, 16163 Genova, Italy. ${ }^{2}$ Nanochemistry Department, Istituto Italiano di Tecnologia, via Morego 30, 16163 Genova, Italy. ${ }^{3}$ Dipartimento di Chimica e Chimica Industriale, Università degli Studi di Genova, Via Dodecaneso 31, 16146 Genova, Italy. ${ }^{4}$ Department of Electrical and Electronic Engineering, University of Cagliari, via Marengo 2, 09123 Cagliari, Italy. ${ }^{5}$ Dipartimento di Fisica, Università degli Studi di Genova, Via Dodecaneso 33, 16146 Genova, Italy. ${ }^{6}$ Materials Characterization Facility, Istituto Italiano di Tecnologia, Via Morego 30, 16163

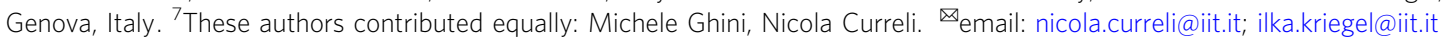


$\mathrm{D}$ oped metal oxide (MO) nanocrystals (NCs) are gaining the attention of the scientific community thanks to their unique properties, such as high electron mobility ${ }^{1}$, the tuneability of their carrier density over several orders of magnitude ${ }^{2}$, chemical stability ${ }^{3}$, and low toxicity ${ }^{3}$, as well as suitable operating temperature ${ }^{1}$, which makes them appropriate for a large plethora of applications, ranging from nanoelectronics and plasmonics to the next-generation energy storage ${ }^{3-11}$. In doped MO NCs, surface states, such as surface trap states, defects, vacancies, as well as surface ligands and other bound molecules induce Fermi level pinning causing an upward bending of the energetic bands ${ }^{2,4,12-18}$. The spatially varying conduction band translates into a gradient in the carrier density $\left(n_{e}\right)$, sufficient to suppress entirely the metallic behavior of carriers close to the nanocrystal surface. This depletion region effectively acts as a dielectric ${ }^{2,12,17,19}$. Hence, the homogeneous flat-band model, which neglects Fermi level pinning, is not sufficient to accurately describe the behavior of free carriers in MO NCs, as introduced by other groups ${ }^{2,17,19,20}$. In fact, the depletion layer formation considerably affects the conductivity of NC films and their plasmonic behavior, with direct implications on the electric field enhancement, the localized surface plasmon resonance (LSPR) modulation and its sensitivity to the surroundings ${ }^{2,17,19}$. Furthermore, the presence of a surface depletion region induces an important alteration to the particle dielectric function ${ }^{2}$.

Given the strong impact of depletion layers on the optoelectronic properties of nanoscale oxide materials, in this work, we aimed at exploiting the depletion layer formation to control energetic band profiles as a means to understand and improve material characteristics. We explore depletion layer engineering beyond surface states by introducing additional electronic interfaces and by dynamically modulating the carrier density via post-synthetic approaches. We experimentally exemplify this scheme with Sn-doped Indium Oxide (ITO)- $\mathrm{In}_{2} \mathrm{O}_{3}$ core-shell NCs and the fine-tuning of the shell thickness $\left(t_{s}\right)$ as well as capacitive charge injection with light (i.e., photodoping). Numerical simulations on both cases serve as a framework to describe in detail the nanoscale evolution of their electronic structure supported by an empirical model that describes the experimental optical properties of all NCs before and after photodoping. The empirical fit model together with electron counting experiments support the band structure calculations well. Through this combined theoretical and experimental work, we unveil that double band bending is a key characteristic of ITO$\mathrm{In}_{2} \mathrm{O}_{3}$ core-shell NCs, describing well also the dynamic introduction of extra electrons via photodoping, a process not fully explained yet. We found that the photo-induced band bending results in an increase in $n_{e}$ predominantly in shell, contradicting the previously reported explanation of a uniform rise of the flatband Fermi level as main mechanism for photodoping 11,16,21,22. Furthermore, the observed band bending supports charge separation towards the NC interface and avoids possible recombination. We finally exploit depletion layer engineering to improve the capacitive charging process in doped metal oxide nanocrystals upon photodoping, resulting in an accumulation of more than 600 stored charges per nanocrystal of the same size.

\section{Results}

We performed numerical simulations based on the solution of Poisson's equation ${ }^{2}$ within the parabolic band approximation to illustrate the band structure of NCs and their depletion layer formation (extended details on the calculations are reported in the Supplementary Information $)^{23,24}$. Here, the depletion layer is defined as the region of the NC where $n_{e}$ drops below $10^{26} \mathrm{~m}^{-3}$ (threshold value at which we can detect plasmonic features) ${ }^{19}$. In Fig. 1 we show the spatially dependent profile of the conduction band as a result of the upward band bending and its effect on the depletion layer width $(W)$ for different parameters, such as surface potentials $\left(E_{S}\right)$ (Fig. 1a), different materials (Fig. 1b) and the introduction of additional electronic interfaces (Fig. 1c and Fig. 1d). In the first case, we consider the effect of surface states on the depletion layer formation. The effect of Fermi level pinning is modeled by a fixed surface potential $\left(E_{S}=0,0.5,1,1.5,2 \mathrm{eV}\right)$, from which the band bending profile is derived. An ITO/surface electronic interface is formed. The value of $E_{S}$ can be found experimentally and it is a peculiar parameter for each material interface. It depends on several factors, such as specific densities of trap states, presence of defects and vacancies as well as surface ligands ${ }^{2}$. For increasing $E_{S}$, we observe an increase in the depletion layer width, which affects a larger fraction of the NC volume (Fig. 1a). These results indicate the importance of surface control to engineer the band structure of NCs. Fig. $1 \mathrm{~b}$ reports the effects of changing the composition of the $\mathrm{NC}$ while keeping $E_{S}$ at a fixed energy. The choice of material, the elemental composition, permittivity $(\varepsilon)$, bandgap energy $\left(E_{g}\right)$ and the control over doping levels are of fundamental importance. Different materials, as in this case $\mathrm{ZnO}, \mathrm{In}_{2} \mathrm{O}_{3}$, ITO and CdO, have a specific impact on the band bending, showing that $W$ is a unique feature of each system. Another powerful parameter to control the depletion layer and the energy level profile is the introduction of additional electronic interfaces beyond the surface of the nanoparticle. One example is the ITO- $\mathrm{In}_{2} \mathrm{O}_{3}$ core-shell nanocrystal system (Fig. 1c).

By surrounding the core ITO NC with $\mathrm{In}_{2} \mathrm{O}_{3}$, two electronic interfaces are formed: ITO/ $\operatorname{In}_{2} \mathrm{O}_{3}$ and $\operatorname{In}_{2} \mathrm{O}_{3} /$ surface. In this case, $E_{S}$ is approximately $0.2 \mathrm{eV}$ below the conduction band minimum of $\mathrm{In}_{2} \mathrm{O}_{3}$, as reported in literature ${ }^{2}$. While in uniform NCs (ITO core only) the band profile is determined by the radial depletion region near the NC surface, the addition of shell layers with thickness $t_{s}$ strongly affects the band's profile ultimately resulting into a double bending of the conduction band. Hence, shell tuning is an effective tool to control $W$ and the shape of the electronic bands inside the NC volume. This effect can be further extended when combining multiple materials sequentially together in coremultishell NC architectures. Figure 1d reports a heterostructure based on three different materials, introducing three electronic interfaces (other combinations of materials and structures are reported in the Supplementary Information, Supplementary Fig. 1). This leads to non-trivial bending and highlights that it is possible to design targeted band structures at the synthesis stage by combining two or more materials in core-shell or core-multishell heterostructures with varying width.

Effective control over NC geometry, size and doping level is crucial to make reliable quantitative assessment of $W$. To experimentally investigate depletion layer engineering predicted by numerical calculations, we synthetized ITO- $\operatorname{In}_{2} \mathrm{O}_{3}$ core-shell NCs with varying shell thickness $t_{s}$ and induced a dynamic variation of their carrier density via photodoping (see Supplementary Information for further details on synthesis methods). Figure $2 \mathrm{a}$ shows the TEM images illustrating the progressive growth of the NC due to the formation of the $\operatorname{In}_{2} \mathrm{O}_{3}$ shell around the ITO core. The crystalline integrity of the samples was confirmed by XRD measurements (Supplementary Fig. 2). We collected multiple aliquots during the synthesis at different stages of the growth resulting in a set of samples with the same physical core size $\left(R_{\text {core }}=5.5 \mathrm{~nm}-\mathrm{C} 0\right)$ and various shell sizes (S1-S5, with $t_{s}=$ $1.15 \mathrm{~nm}, 1.9 \mathrm{~nm}, 2.9 \mathrm{~nm}, 4.25 \mathrm{~nm}$ ). The successful achievement of core-shell structures was confirmed by a comparison of the Sn-dopant concentrations obtained by two different techniques: inductively coupled plasma mass spectrometry (ICP-OES) as volume sensitive technique and X-ray Photoelectron Spectroscopy (XPS) as a surface sensitive technique (Fig. 2b). These techniques probe the volumetric and surface content of $S n$ atoms, respectively, and have been shown to be effective methods in 

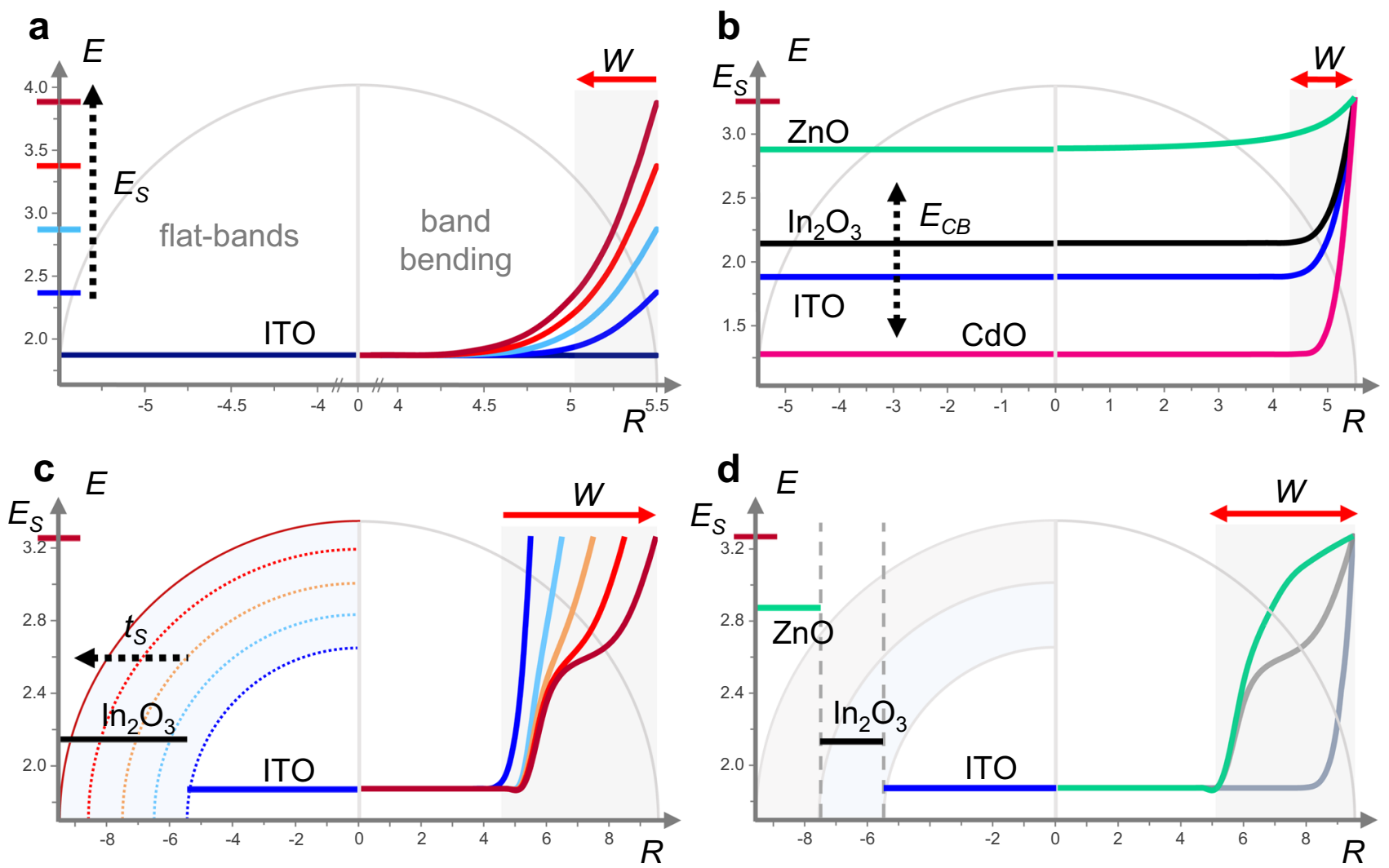

Fig. 1 Depletion layer engineering of metal oxide NCs via tuning of structural and electrical properties. The morphology of the nanocrystal (NC) is illustrated by a semi-circle with its extension $R$ in the abscissa (in $\mathrm{nm}$ ) and the energy in the ordinate (in eV). In each panel, the left side of the sphere represents the flat-band potentials in the non-equilibrium condition, while the right side reports the band bending due to the Fermi level pinning (with the shaded region indicating the depletion width, $W$ ). Band profiles were calculated by numerically solving the Poisson's equation. a Tuning of surface state potentials $\left(E_{S}\right)$. Increasing the $E_{S}$ results in the expansion of the depletion width, $W$ (progressively from blue to red). $\mathbf{b}$ Impact of different materials on $W$ at fixed $E_{S}$. c Expansion of $W$ and double bending of the depletion layer in a core-shell structure of ITO- $\ln _{2} \mathrm{O}_{3}$ with a core radius $\left(R_{\text {core }}\right)$ of $5.5 \mathrm{~nm}$ and varying shell thickness ( $t_{s}=0,1,2,3,4 \mathrm{~nm}$, i.e., blue, light blue, orange, red and dark red, respectively). d Multiple shell system by combining an ITO core $\left(R_{\text {core }}=5.5 \mathrm{~nm}\right)$ with a $\mathrm{n}_{2} \mathrm{O}_{3}$ and $\mathrm{ZnO}$ shell, with total radius $R=9.5 \mathrm{~nm}$. The band shows a complex profile with a triple bending (green curve). The gray curves illustrate the previously reported case of a uniform ITO NC (dark gray) and an ITO- $\ln _{2} \mathrm{O}_{3}$ core-shell NC (light gray) with total radius $R=9.5 \mathrm{~nm}$ for comparison.

elucidating nanocrystal dopant distributions ${ }^{25}$. We observe a higher Sn-concentration from the volume-sensitive measurements (black curve in Fig. 2b) as compared to the surfacesensitive measurements (red curve in Fig. 2b) in all samples with shell. This indicates that the Sn dopants are localized in the core of the NCs without significant diffusion of Sn atoms into the shell (further analysis on diffusion effects can be found in Supplementary Fig. 3). The absorption spectra of the representative samples, normalized to the maximum are reported in Fig. 2c (dotted curves). The spectra are governed by intense resonances in the near-infrared (NIR) that are assigned to localized surface plasmon resonances (LSPRs) as a result of free electrons in the highly doped semiconductor (typically in the range of $\left.10^{27} \mathrm{~m}^{-3}\right)^{2,4,25-27}$. The LSPR peak position $\omega_{\text {LSPR }}$ and its peak profile are correlated to several factors, such as the NC geometrical features (e.g., $\left.R_{\text {core }}, t_{s}\right), n_{e}$, the depletion layer width $(W)$, the dielectric constant of the surrounding medium $\left(\varepsilon_{m}\right)$, as well as the structural defects and dopant concentration, providing a unique spectral signature of such parameters ${ }^{21,28-30}$. We will come back to this point later when describing the empirical fit model. From the modulation of the LSPR upon shell growth, we observe an initial blue shift of the LSPR (see Supplementary Information, Supplementary Fig. 4). This is ascribed to the activation of surface dopants with the growth of a thin $\operatorname{In}_{2} \mathrm{O}_{3}$ layer, which results in an increased carrier density ${ }^{25}$. The following continuous red shift of the LSPR is due to the presence of an increasing shell thickness $t_{s}$ that modifies the dielectric surrounding of the $\mathrm{NC}^{25}$. Notably, in particles with a critical thickness $t_{s}^{*}=2.7 \mathrm{~nm}$, a second shoulder appears in the spectrum. This indicates a more complex carrier density profile within the core-shell nanocrystals which induces an independent resonating mode, generated by a sufficiently high carrier density in the shell of the nanoparticle ${ }^{31}$.

To further study the electronic structure of core-shell NCs out of the equilibrium conditions, we post-synthetically alter the number of free carriers via photodoping $4,11,21,32$. Photodoping consists of introducing multiple free charge carriers via light absorption and suppressing carrier recombination by the quenching of the holes with hole scavengers ${ }^{11,21,33}$. The photodoping process in colloidal NCs has been recently investigated with optical and electrochemical (e.g., potentiometric titration) measurements ${ }^{4,11,21}$. Here, we induce the photodoping by exposing our colloidal NCs to light beyond the ITO band-gap in the ultraviolet (UV) region $(300 \mathrm{~nm}-4.1 \mathrm{eV}, \mathrm{FWHM}=20 \mathrm{~nm})$ and an intensity of $36.8 \mathrm{~mW} \mathrm{~cm}^{-2}$. Figure $2 \mathrm{c}$ shows the normalized absorbance of three representative examples before (dotted curves) and after (solid curves) the exposure to $20 \mathrm{~min}$ of UV light. After the introduction of extra photocarriers into the system the LSPR absorption increases in intensity $\left(\Delta I=I^{\text {photodoped }}-I^{\text {as synthesized }}\right)$ and its energy shifts $\left(\Delta \omega=\omega_{L S P R}^{\text {photodoped }}-\omega_{L S P R}^{\text {as synthesized }}\right)$. The photoinduced effects progressively appear with the amount of light 
a
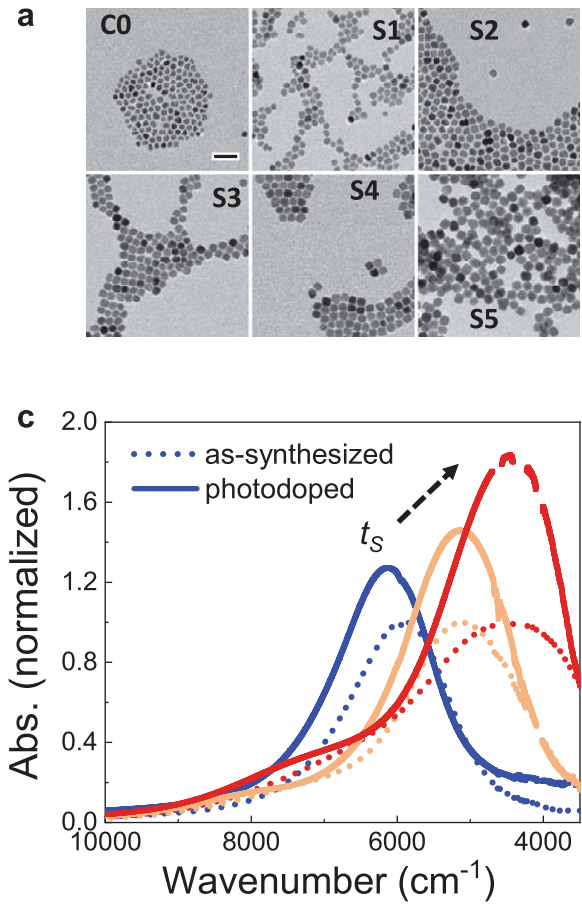

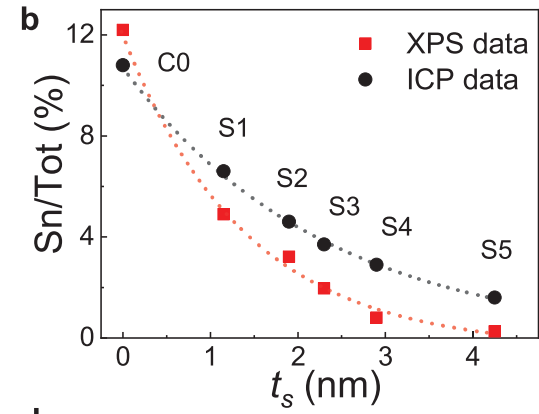

d

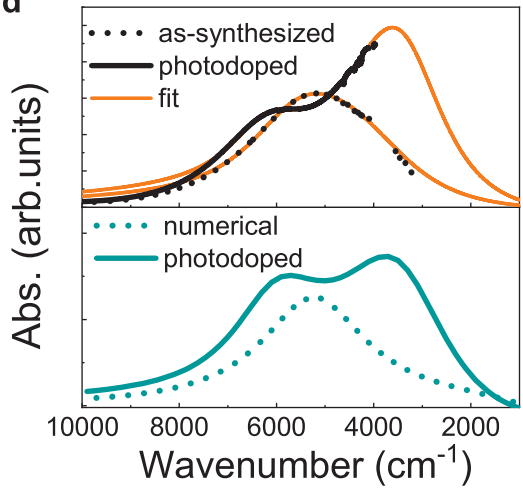

Fig. 2 ITO- $\mathbf{I n}_{\mathbf{2}} \mathbf{O}_{\mathbf{3}}$ core-shell colloidal nanocrystals. a TEM images of the nanocrystals at different stages of the synthesis. Starting from an aliquot of the core (CO), layers of $\ln _{2} \mathrm{O}_{3}$ progressively form a thicker shell around the ITO nuclei (S1-S5). Scale bar is $50 \mathrm{~nm}$ for each panel. $\mathbf{b}$ Comparison between the Sn-dopant concentrations obtained from inductively coupled plasma mass spectrometry (ICP-OES) and X-ray Photoelectron Spectroscopy (XPS) as a function of shell thickness $\left(t_{s}\right)$. The higher $\mathrm{Sn}$ /Tot values of the volume sensitive ICP-OES measurements with respect to the surface sensitive XPS measurements indicate that Sn atoms remain segregated in the core of the NCs. Dashed lines are to guide the eye. c Normalized absorbance of typical ITO$\mathrm{In}_{2} \mathrm{O}_{3}$ core-shell samples with increasing shell size, in as-synthesized (dotted lines) and photodoped (solid lines) cases, with a $t_{s}$ of $0.15 \mathrm{~nm}$ (blue), $1.85 \mathrm{~nm}$ (orange), and $2.3 \mathrm{~nm}$ (red). Growing an undoped shell continuously redshifts the energy of the localized surface plasmon resonance (LSPR) peak in the NIR. After 20 min of UV exposure, the intensity of the LSPR peak increases significantly, reaching values up to almost double its initial absorbance. $\mathbf{d}$ Top panel: Absorbance of sample S5 ( $t_{s}=4.25 \mathrm{~nm}$ ), as-synthesized (dotted line) and photodoped (solid line). The fitting of the experimental data using the multi-layer optical model is depicted by the orange lines. Bottom panel: Numerical simulations of the absorbance of the same ITO-I ${ }_{2} \mathrm{O}_{3} \mathrm{NC}$ with $t_{s}=$ $4.25 \mathrm{~nm}$ (dotted green lines) and the simulation with extra electrons (i.e., photodoping, solid green line).

absorbed (Supplementary Fig. 5) in agreement with previous reports in the literature $4,16,21,34$. The introduced photoelectrons add to the initial free carrier density leading to a stronger interaction with the incoming radiation and hence an increased LSPR absorption. The impact of the photodoping on the LSPR modulation is extremely sensitive to $t_{s}$, with $\Delta I$ almost doubling in the case of the biggest NCs. In Fig. 2d, the normalized absorption spectra for the sample having a $t_{s}=4.25 \mathrm{~nm}$ are shown before (black dotted curve) and after (black continue curve) photodoping. In this case, it is possible to note a particularly strong splitting of the LSPR into two major contributions. These results display an enhanced sensitivity of the LSPR peak to photodoping by increasing $t_{s}$ and indirectly hint towards an increased number of stored photoelectrons for higher $t_{s}$ (since the LSPR absorption is proportional to $\left.n_{e}^{2 / 3}\right)^{19}$.

We now investigate the same system of ITO- $\operatorname{In}_{2} \mathrm{O}_{3} \mathrm{NCs}$ with varying thickness $t_{s}$ with numerical methods as introduced above. The values for $t_{s}$ were chosen equivalent to the size of synthesized NCs. To further investigate also the photodoping process, we numerically calculate the effects of additional free electrons in the system as a function of $t_{s}$. To this aim, we introduced a generation function $G(R)=I_{0} \alpha \beta e^{-\alpha R}$, which extends the Poisson's equation by an additional term that represents the spatial distribution of the extra free carriers introduced into the system via photodoping. The intensity of incident photon flux is reported as $I_{0}, \alpha$ denotes the photon absorption coefficient, and $\beta$ denotes the quantum efficiency, respectively ${ }^{35}$. We target to identify how their presence modifies the energy bands and carrier density distribution of the system. In this way, we go beyond the results introduced in Fig. 1 and we assess the dynamic, post-synthetic variation of the electronic band profiles via light-induced charge injection, i.e., photodoping. A comparative study reporting electronic structures and carrier density profiles both before and after photodoping are shown in Fig. 3a and Fig. 3b. We first discuss the effects of shell formation on the electronic structure of the NCs (black curves in Fig. 3a). The Fermi level pinning anchors the depleted region to the surface of the nanocrystal at the same energy, irrespective of $t_{s}$. However, with increasing $t_{s}$ it affects more strongly the $\operatorname{In}_{2} \mathrm{O}_{3}$ shell region, which effectively shields the ITO core from depletion. Consequently, even if $W$ increases, the depletion layer progressively shifts towards the outer region of the NC. An intermediate region between core and surface states is, thus, introduced, resulting into the expansion of the active core region $\left(R_{\text {active }}\right)$, i.e., the region of the NC volume not affected by $W$, which is typically larger than $R_{\text {core }}$. In fact, the spatial extent of these electronic features does not correspond to the as synthesized structural parameters (i.e., $R_{\text {core }}, t_{s}$ ). This expansion is not due to an introduction of extra donor atoms, nor to diffusion effects (more details in Supplementary Information, Supplementary Fig. 3). With increasing $t_{s}$, a more pronounced bending of the bands occurs, and it extends for nanometers into the NC. The corresponding carrier density distribution (black curves in Fig. 3b) shows a non-trivial profile. The double bending can be explained by a leakage of carriers into the shell. The carrier density in extended regions of the undoped shell reaches values beyond $\sim 1 \cdot 10^{26} \mathrm{~m}^{-3}$. The presence of carrier density in this 

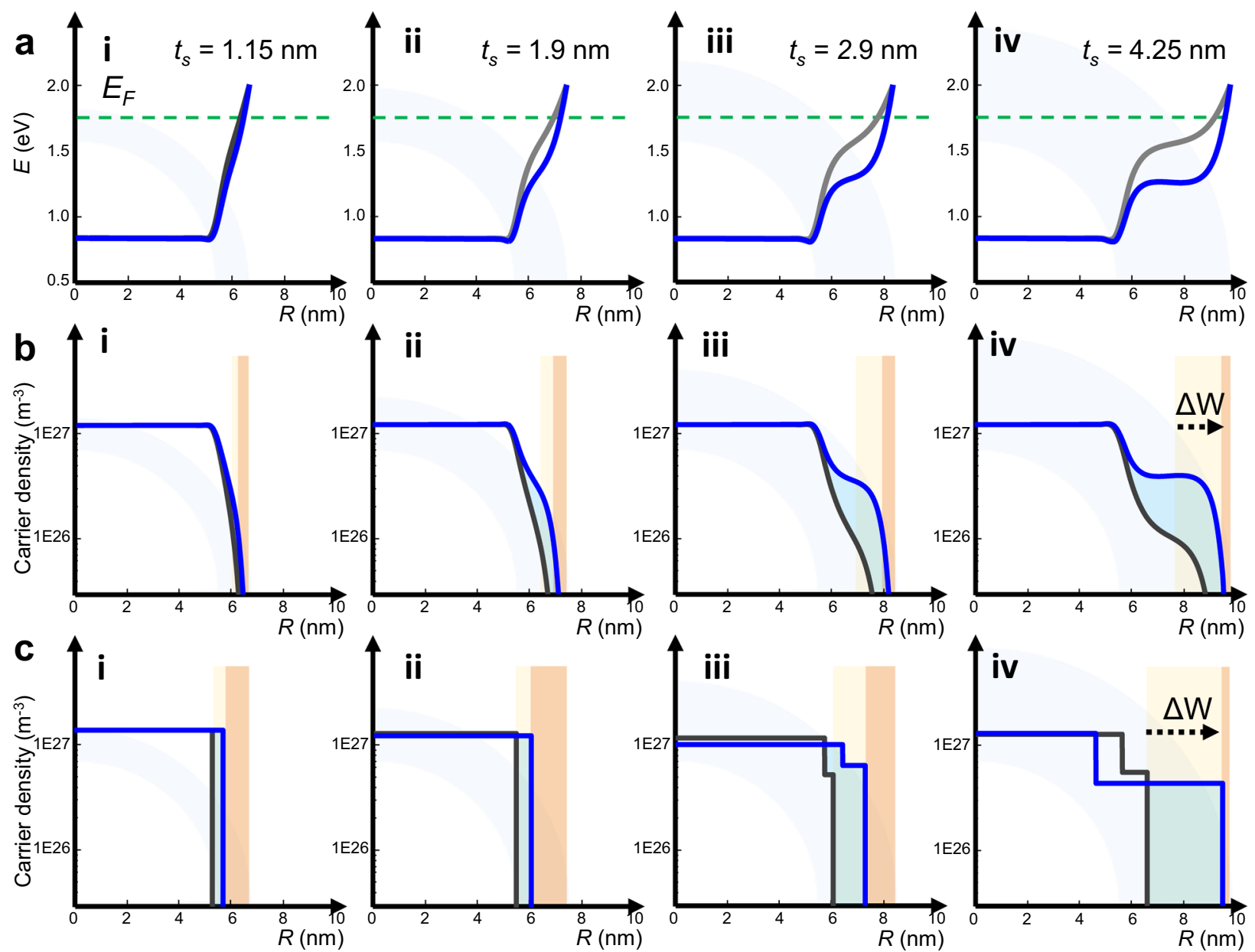

Fig. 3 Band profiles and depletion layer modulation via photodoping. a Simulated conduction band profiles of core-shell nanocrystals with increasing shell thickness before (black curve) and after (blue curve) the injection of photoelectrons. The light-induced bending of the bands close to the surface of the nanocrystal is the main mechanism responsible for the storage of extra electrons. $\mathbf{b}$ Calculated electron density profiles of the same nanocrystals. The depletion region $W$ is shaded in orange. The active region $\left(R_{\text {active }}\right)$ of the NC is observed as the region of the NC volume not affected by $W$. A discrepancy between $R_{\text {active }}$ and the structural core $\left(R_{\text {core }}\right)$ and shell $\left(t_{s}\right)$ dimensions (given in white and light blue in the background) is observed. The largest variations in $n_{e}$ after photodoping occur towards the edge region of the nanocrystal with a significant rise of carrier density in the shell region (blue shaded regions). Due to photodoping, the active region expands $\left(\Delta R_{\text {active }}>0\right)$. Consequently, the depletion layer is progressively suppressed $(\Delta W<0)$ during the storage of electrons with photodoping (from light orange to dark orange). c Carrier density profiles obtained from the fitting of the experimental data using the multilayer optical model. The expansion of the active region $\left(R_{\text {active }}\right)$ and $W$ modulations of core-shell NCs of increasing $t_{s}$ upon photodoping are observed similar to from the results from the numerical simulations. The filling of the depleted regions with extra electrons is highlighted in blue in panels $\mathbf{b}$ and $\mathbf{c}$ The pale blue circle represents the NC shell.

range in the undoped $\operatorname{In}_{2} \mathrm{O}_{3}$ region indicates that for $t_{s}>t_{s}^{*}=$ $2.7 \mathrm{~nm}$ it is not appropriate to approximate the ITO- $\mathrm{In}_{2} \mathrm{O}_{3}$ system as a doped core-dielectric shell. Instead, it must be considered as a dual-plasmonic material with a specific carrier density in the core $\left(n_{\text {core }}=1.1 \cdot 10^{27} \mathrm{~m}^{-3}\right)$ and enhanced carrier density in the shell with $n_{\text {shell }}<n_{\text {core }}$. This explains the experimentally observed double features in the LSPR (see Fig. 2c), which are reproduced by our simulated absorption spectra (Fig. $2 \mathrm{~d}$ ) when implementing the carrier density profile extracted from Fig. 3b. The observed double bending of the energetic bands becomes more pronounced upon photodoping in all samples (blue curves in Fig. 3a) with an immediate impact on the carrier density distribution (Fig. 3b). Indeed, after photodoping for samples beyond $t_{s}^{*}$ the band profile approaches a step function with two distinct energy levels: the conduction band (CB) level in the core and an energetic level approx. $0.45 \mathrm{eV}$ above the $\mathrm{CB}$ in the shell. This effect is observed in the carrier density profile as a (close to) two-step profile. In the samples with $t_{s}>t_{s}^{*}=2.7 \mathrm{~nm}$ the maximum $n_{e}$ reached in the NC shell differs from the one in the core, reaching values of around $\sim 4 \cdot 10^{26} \mathrm{~m}^{-3}$, while the core carrier density remains nearly constant. The light-induced modulation of the depletion layer width $\left(\Delta W=W^{\text {photodoped }}-W^{\text {as synthesized }}\right)$ increases with a $\Delta W \sim t_{s}{ }^{3}$ law (Supplementary Fig. 6). Since the photo-generated extra carriers tend to fill $W$, larger $\Delta W$ values of the assynthesized NCs justify the possibility to store more electrons in NCs with bigger shells. Hence, from our simulations we conclude that the filling of electronically depleted regions is the main mechanism behind the photodoping process of metal oxide NCs. These findings seem to be in contradiction with the literature reports on the experimentally observed uniform rise of the Fermi energy level as a result of the photo-induced accumulation of multiple photoelectrons, as shown by Schimpf et al. ${ }^{11,16,21,22}$. However, the plasmonic effects of double features observed after photodoping of core-shell nanocrystals are not explainable with a simple Fermi level rise. In a flat-band scenario a uniform rise of Fermi level would necessary imply a blue-shift of $\omega_{L S P R}$, while we experimentally observed photodoped NCs with no blue-shift, a red shift (see Fig. $4 \mathrm{~b}$, below) or even a splitting of the $\omega_{L S P R}$ 

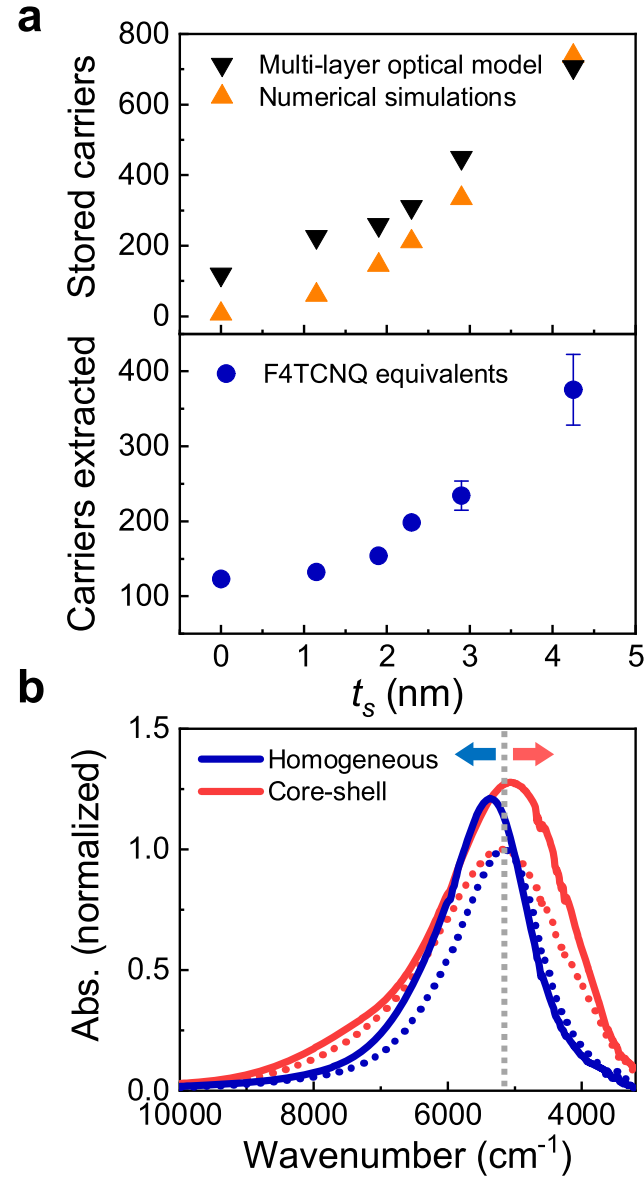

Fig. 4 Charge storage enhancement through depletion layer engineering. a Upper panel: Photoelectrons stored in the NCs as a function of the shell thickness, showing the $\Delta N_{e} \sim t_{s}{ }^{3}$ trend for values extracted from the empirical multi-layer model (black curve) and the numerical simulations (orange curve). Bottom Panel: Number of electrons extracted via chemical titration using F4TCNQ molecules as a function of $t_{s}$. The error bars represent the lower and upper limit of the charges extracted within the uncertainty of the data. $\mathbf{b}$ Experimental comparison between the optical response of two samples with same size and doping concentrations but different electronic structure, before (dotted line) and after (solid line) photodoping (homogeneous ITO in blue, core-shell ITO- $\mathrm{In}_{2} \mathrm{O}_{3}$ in red). The sensitivity of the LSPR modulation via photodoping is enhanced in the core-shell case. We highlight that the peak position of the LSPR after photodoping blueshifts in the homogeneous case, while it redshifts in the core-shell case indicating that depletion layer modulation is the main process of photodoping (see discussion above).

(Fig. 2d) in the core-shell samples. Hence, we hypothesize that the experimentally observed uniform rise of the Fermi level, as observed by Schimpf et al. ${ }^{11,16,21,22}$ is a result of the averaging over the local carrier densities in different fractions of the NC and in particular in the near surface regions.

To further test our theory, we approach the photodoping process by applying an empirical model to fit the spectra of each sample. Representative fits are shown as orange curves in Fig. 2d. The plasmonic properties of doped MO NCs can be well described within the framework of the Mie scattering theory in the quasistatic approximation. For our samples, we found that the classical Drude model is sufficient to accurately describe the plasmon response, with quantum effects representing only a minor correction. However, for small NCs (e.g., $R<4 \mathrm{~nm}$ ), and low free charge regime (e.g., $N_{e}<100$ ), quantum mechanical effects cannot be neglected ${ }^{36}$ (further discussion in the Supplementary Information). The optical response of metals and heavily-doped semiconductors is characterized by the polarizability of the free electrons, depicted in the Drude-Lorentz model with the complex dielectric permittivity $\varepsilon(\omega)=\varepsilon_{\infty}-\omega_{P}^{2} /\left(\omega^{2}+i \omega \Gamma\right)$. Here, the bulk plasma frequency $\omega_{p}=\sqrt{n_{e} e^{2} / \varepsilon_{0} m^{*}}$ is a function of the free carrier density $\left(n_{e}\right)$ and the effective electron mass $\left(m^{*}\right), \Gamma$ is a damping parameter accounting for electron-electron scattering and $\varepsilon_{\infty}$ is the high frequency dielectric constant. Within this picture, $\omega_{\text {LSPR }}$ is directly linked to $\omega_{p}$ of the material. The tuneability of the LSPR is provided by the proportionality to $n_{e}$, which is related to the number of free charges over the active volume $\left(n_{e} \sim N_{e} / R_{\text {active }}^{3}\right)$. Hence, we can link the absorption, which is our physical observable, to the electronic structure of the system. In previous works, the effect of depletion layers was addressed by Zandi et al., who introduced an effective dielectric function using a Maxwell-Garnett effective medium approximation (EMA) ${ }^{2}$. This approach shows that accumulating charges in the $\mathrm{NC}$ as a result of electrochemical doping has the effect of increasing the intensity and shifting the position of the LSPR peak as a result of the varying $W^{2}$. We adapt this model by implementing a core-shell structure with frequency dependent core dielectric function $\varepsilon_{\text {core }}(\omega)$ (and constant carrier density, $n_{\text {core }}$ ) surrounded by a dielectric shell with $\varepsilon_{D L}=4$ in the depletion layer. Outside the sphere a dielectric medium with fixed $\varepsilon_{m}=2.09$ is present. Within this picture, we approximate the continuous carrier density profile $n_{e}(R)$ with discrete regions of uniform density, while we define $n_{e}=0$ inside the depletion region (Fig. 3c). We found that the two-layer model describes well the optical spectra when $t_{s}<t_{s}^{*}=2.7 \mathrm{~nm}$ (Fig. $3 c$, i and ii). Importantly, for $t_{s}>t_{s}^{*}$ and most photodoping cases, we found that it was necessary to extend this model in order to fit the spectra. To this aim, we developed a threelayer model based on the Maxwell-Garnett EMA with three concentric regions. In the first two regions the inner core and first shell region, which sum up to $R_{\text {active }}$, have a frequency dependent dielectric constant of $\varepsilon_{\text {core }}(\omega)$ and $\varepsilon_{\text {shell }}(\omega)$ with constant carrier densities of $n_{\text {core }}(R)$ and $n_{\text {shell }}(R)$, respectively. Surrounding the frequency dependent core and shell dielectric functions is an additional layer that accounts for the depletion of carriers in the shell, which was not previously considered in models found in literature $2,28,31$. Hence, these two concentric regions are surrounded by a third depleted layer of thickness $W$ with fixed $\varepsilon_{D L}=4$ and zero carrier density. The surrounding dielectric medium is $\varepsilon_{m}=2.09$. By taking into account the formation of an additional depletion layer due to the electronic interface between shell and surface, our model goes beyond what has been implemented so far to describe capacitive charges in $\mathrm{MO} \mathrm{NCs}^{2,28,31}$. In our study, for all values of $t_{s}$, the most notable changes in $n_{e}$ after photodoping are observed to effectively increase $R_{\text {active }}$ and decrease $W^{28}$. The core carrier density $n_{\text {core }}$ remains nearly constant with variations of less than $14 \%$, while a significant variation occurs in the shell regions, with $n_{\text {shell }}$ around $\sim 5.4 \cdot 10^{26} \mathrm{~m}^{-3}$.

We give a quantitative comparison between the numerical and empirical approach by plotting the amount of stored carriers in the NC $\left(\Delta N_{e}\right)$, defined as the difference between the free carriers of the photodoped NC and the as-synthesized NC $\left(\Delta N_{e}=N_{e}^{\text {photodoped }}-N_{e}^{\text {as synthesized }}\right)$. We observe a good agreement between both approaches, finding that $\Delta N_{e}$ increases with $t_{s}$ with a $\Delta N_{e} \sim t_{s}^{3}$ trend, reaching values as high as 600 extra electrons (Fig. 4a). We advanced the studies of the NC stored carriers by using titration on photodoped NCs to count the number of stored electrons (further details in the Supplementary Information) $)^{4,11,37}$. By using molecular oxidants (F4TCNQ) to titrate the electrons, we directly measure the average number of electrons extracted per NC. F4TCNQ in this study acts as an electron acceptor. The optical features of F4TCNQ hold as a signature to quantify the extracted electrons. We observe an increase of the number of extracted 
photocarriers with increasing $t_{s}$, in agreement with the trend reported for numerical simulations and empirical modeling (Fig. 4a). The discrepancy, up to a factor 2 in the case of large core-shell NCs, is most probably related to a reduced efficiency in the carrier extraction process. Nevertheless, the $\Delta N_{e} \sim t_{s}{ }^{3}$ trend is reproduced displaying that the electron counting experiments together with the empirical fit model support the band structure calculations well.

Finally, we aim at isolating the impact of depletion layer engineering from the volume dependence of $\Delta N_{e}$ as shown from Schimpf et al. ${ }^{16}$. From numerical simulations, we found that the number of stored carriers in NCs with a core-shell architecture are significantly larger than in the pure ITO case (Supplementary Fig. 7a and Supplementary Fig. 7b). To confirm this result experimentally, we perform a quantitative analysis of one specific $t_{s}$ and compare it to a similar NC without shell (only core) with all other parameters unchanged (i.e., total NC radius $R$, doping density $N_{d}$, experimental conditions). The optical absorption spectra before and after photodoping are depicted in Fig. 4b. By applying our empirical model to this case, we obtain that core-shell NCs can accumulate $\sim 40 \%$ more carriers than uniform ITO NCs of the same size. Our numerical simulations predict that this enhancement increases with increasing shell thickness. Hence, we demonstrate that depletion layer engineering can improve charge storage capacity and more generally that the band bending delivers an additional degree of freedom to artificially engineer the optoelectronic properties of $\mathrm{MO}$ NCs, both during synthesis and post-synthetically.

\section{Discussion}

In this work, we demonstrate that depletion layer engineering is an important tool to design and control energetic band profiles in metal oxide NCs. Our results are based on a combination of theory and experiment: we implement a numerical model that is able to account for additional free carriers in the MO NCs, we developed an empirical three-layer model that describes the optical response of the (photodoped) core-shell ITO- $\mathrm{In}_{2} \mathrm{O}_{3} \mathrm{NCs}$, and confirmed our results with experimental electron counting experiments through reaction with F4TCNQ. From this combined theoretical and experimental approach, we found that, first, double bending of the bands dominates the electronic structure of (photodoped) core-shell ITO- $\mathrm{In}_{2} \mathrm{O}_{3} \mathrm{NCs}$ and that the depletion layer predominantly affects the $\operatorname{In}_{2} \mathrm{O}_{3}$ shell. Second, the electronic rearrangement of energy bands and the filling of electronically depleted regions resulting in the evolution of different levels of carrier density in core and shell are the main mechanism behind the photodoping process of metal oxides NCs. Third, depletion layer engineering allows enhancing the charge storage capability of ITO NCs of the same size. We can extend this model to other systems as well demonstrating the validity of our approach. Our results show that the modulation of the depletion layer represents an interesting avenue to design and improve the properties of MO NCs and their core-shell or core-multishell structures. We foresee multiple practical applications ranging from energy storage to sensing for ITO- and similar metal oxide nanocrystalsbased devices that will benefit from the control of electronic band profiles through depletion layer engineering.

\section{Methods}

Core-shell nanocrystal synthesis. ITO/ $\mathrm{In}_{2} \mathrm{O}_{3}$ core/shell nanocrystals were synthesized in a continuous growth approach with the following step-by-step procedure $19,25,38$. A precursor solution was prepared mixing in a flask tin(IV) acetate and indium(III) acetate in a 1:9 Sn:In ratio. Subsequently, oleic acid was added in a 1:6 metal to acid ratio to yield a $10 \%$ Sn doped ITO precursor solution. The flask was stirred and left at $150{ }^{\circ} \mathrm{C}$ under $\mathrm{N}_{2}$ for $3 \mathrm{~h}$ for degassing The ITO nanocrystals (core) were first prepared by adding the ITO precursor solution via a syringe pump (drop-by-drop at a rate of $0.35 \mathrm{~mL} / \mathrm{min}$ ) to $13.0 \mathrm{~mL}$ of oleyl alcohol at $290^{\circ} \mathrm{C}$. During the slow-injection procedure a flow of $130 \mathrm{~mL} / \mathrm{min}$ of $\mathrm{N}_{2}$ gas was kept in the reaction flask to quickly remove any water vapor formed during the reaction. The ITO cores, stabilized with oleic acid ligands, were continuously grown to a size of $5.5 \mathrm{~nm}$ (radius) and isolated by precipitating with $12 \mathrm{~mL}$ ethanol The solid part was collected by centrifugation at $7300 \mathrm{rpm}(5540 \times \mathrm{g})$ for $10 \mathrm{~min}$, washed twice more with ethanol and dispersed in hexane.

Then, part of the cores was kept for analysis and the rest of the solution reintroduced in fresh oleyl alcohol. For shelling, a second precursor solution was prepared by following the same procedure. In order to yield an undoped indium oleate precursor solution, indium(III) acetate was mixed with oleic acid in a 1:6 molar ratio. Undoped indium oleate was added with the same slow-injection procedure described above. Core-shell samples were then washed with ethanol and the process repeated several times until a final size of $\sim 10 \mathrm{~nm}$ (radius) was reached. All experiments were performed on samples collected at different stages of the shell growth, and hence sharing the very same ITO core.

Structural characterization of core-shell NCs. The structural characterization of the samples with different shell thickness were analyzed by transmission electron microscopy (TEM) to determine the size and confirm the successful formation of nanocrystals. TEM measurements were performed with a JEOL JEM-1400Plus operating at $120 \mathrm{kV}$ and using lacey carbon grids supported by a copper mesh. The size distribution of the NCs was extracted using ImageJ tools on the images collected ${ }^{39}$

X-ray Diffraction (XRD) analyses were carried out on a PANanalytical Empyrean X-ray diffractometer equipped with a $1.8 \mathrm{~kW} \mathrm{Cu} \mathrm{Ka} \mathrm{ceramic} \mathrm{X-ray} \mathrm{tube}$ and a PIXcel3D $2 \times 2$ area detector, operating at $45 \mathrm{kV}$ and $40 \mathrm{~mA}$. Specimens for the XRD measurements were prepared by dropping a concentrated NCs solution onto a zero-diffraction silicon substrate. The diffraction patterns were collected under ambient conditions using a parallel beam geometry and the symmetric reflection mode. XRD data analysis was carried out using the HighScore 4.1 software from PANalytical.

X-ray Photoemission Spectroscopy (XPS) measurements were performed on a Kratos Axis Ultra ${ }^{D L D}$ spectrometer, using a monochromatic $\mathrm{Al} \mathrm{Ka}$ source $(15 \mathrm{kV}$ $20 \mathrm{~mA}$ ). Specimens were prepared by dropping a concentrated NCs solution onto a highly ordered pyrolytic graphite (HOPG, ZYA grade) substrate. High resolution spectra of the $\mathrm{Sn} 3 d$ and In $3 d$ regions were acquired at pass energy of $10 \mathrm{eV}$, and energy step of $0.1 \mathrm{eV}$, over a $300 \times 700$ microns area. The photoelectrons were detected at a take-off angle of $\phi=0^{\circ}$ with respect to the surface normal. The pressure in the analysis chamber was maintained below $7 \times 10^{-9}$ Torr for data acquisition. The data was converted to the VAMAS format and processed using the CasaXPS software, version 2.3.24 ${ }^{40}$. The binding energy (BE) scale was internally referenced to $\mathrm{C} 1 s$ peak $(\mathrm{BE}$ for $\mathrm{C}-\mathrm{C}=284.8 \mathrm{eV}$ ). For the quantitative analysis, the areas of In $3 d$ and $\mathrm{Sn} 3 d$ peaks were calculated after applying the appropriate background correction across the binding energy range of the peaks of interest. The relative atomic concentrations were then estimated, using the so-called relative sensitivity factors (RSF) provided by Kratos $\left(\mathrm{RSF}_{\mathrm{In} 3 \mathrm{~d}}=7.265, \mathrm{RSF}_{\mathrm{Sn} 3 \mathrm{~d}}=7.875\right)$

Inductively coupled plasma mass spectrometry (ICP-OES) was performed on all samples to estimate the doping levels and concentrations of the ITO NCs. The elemental analysis was carried out via inductively coupled plasma optical emission spectroscopy (ICP-OES) on an iCAP 6000 Series ICP-OES spectrometer (Thermo Scientific). In a volumetric flask, each sample was dissolved in aqua regia $[\mathrm{HCl} /$ $\left.\mathrm{HNO}_{3} 3: 1(\mathrm{v} / \mathrm{v})\right]$ and left overnight at RT to completely digest the NCs. Afterward, Milli-Q grade water $(18.3 \mathrm{M} \Omega \mathrm{cm})$ was added to the sample. The solution was then filtered using a polytetrafluorethylene membrane filter with $0.45 \mu \mathrm{m}$ pore size. All chemical analyses performed by ICP-OES were affected by a systematic error of about $5 \%$.

Optical measurements. Optical measurements were carried out on a Cary5000 UV-vis-NIR Spectrometer. Spectra were collected in anhydrous toluene in the range $280-3200 \mathrm{~nm}$ with a scan resolution of $1 \mathrm{~nm}$. After drying the solvent, ITO NCs were transferred in anhydrous toluene (Sigma-Aldrich) in a nitrogen filled glove box. Rectangular anaerobic cuvettes with a sealed screw cap (Starna Scientific) were used for photodoping and titration experiments.

Photodoping process. Before photodoping, the ITO- $\mathrm{In}_{2} \mathrm{O}_{3}$ NCs were dissolved in anhydrous toluene, as described above. Subsequently, the photodoping process on the NCs is achieved by illuminating the quartz cuvette containing the solution with the NCs with a UV LED (central wavelength: $300 \mathrm{~nm}$, bandwidth: $20 \mathrm{~nm}$ ). The cuvette was placed at a distance of $12 \mathrm{~mm}$ from the cuvette window (Thorlabs M300L4). UV power density at the front window of the cuvette was $36.8 \mathrm{~mW} \mathrm{~cm}^{-2}$.

Redox titration. The titrant was prepared by dissolving $0.34 \mathrm{mg}$ of F4TCNQ (2,3,5,6-tetrafluoro-7,7,8,8-tetracyanoquinodimethane) in $30 \mathrm{~mL}$ of anhydrous toluene. The NCs solution was prepared in anhydrous toluene and photodoped in the same manner as described above (typical concentration $\sim 0.1 \cdot 10^{-9} \mathrm{~mol} / \mathrm{L}$ ). The titrant addition steps were carried out in the inert environment of a nitrogen-filled glove box to avoid any contact with ambient oxygen. Electron counting was performed after photodoping by spectroscopic analysis of the neutral, anion and dianion forms of the F4TCNQ molecules ${ }^{37}$. In details, the amount of F4TCNQ $\left(n_{F 4 T C N Q}\right.$, in moles) added at each step of the experiment was calculated from volume of titrant introduced 
in the cuvette $(V)$, the titrant concentration $(C=0.085 \mathrm{mg} / \mathrm{mL})$, and the titrant molecular mass $(276.15 \mathrm{~g} / \mathrm{mol}): n_{F 4 T C N Q}(V)=C V / 276.15$. The number of NCs present in solution $\left(n_{N C}\right)$ was calculated from the mean NC size (from TEM images) and the average weight of a NC (from ICP-OES measurements). Thus, the number of F4TCNQ molecules reacted per NC was calculated as: $n_{\text {reacted }}(V)=n_{F 4 T C N Q}(V) / n_{N C}$. To calculate the number of extracted electrons, $n_{\text {reacted }}$ was then multiplied to a factor one or two according to the kind of reaction involved, corresponding to the formation of dianion or anion species, respectively. Two volumes were identified: $V_{1}$, corresponding to the saturation of the dianion reactions (with exclusively two-electron transfers occurring), and $V_{2}$, corresponding to the appearance of neutral peaks signatures (signaling the presence of non-reacted titrants). The growth of anion peaks (i.e., transfer of one electron) between $V_{1}$ and $V_{2}$ indicate that electron transfer reactions keep occurring after $V_{1}$. In this study, two-electron transfers were considered up to the midpoint between $V_{1}$ and $V_{2}\left(V_{\text {mid }}=\left(V_{1}+V_{2}\right) / 2\right.$ and the transfer of one extra electron was considered between $V_{\text {mid }}$ and $V_{2}$. The total number of extracted charges was estimated as $2 e^{-} \cdot n_{\text {reacted }}\left(V_{\text {mid }}\right)+1 e^{-} \cdot\left(n_{\text {reacted }}\left(V_{2}\right)-n_{\text {reacted }}\left(V_{\text {mid }}\right)\right)$. Error bars are representative to the distance between $V_{1}$ and $V_{2}$. The effects of titrants on assynthesized ITO- $\operatorname{In}_{2} \mathrm{O}_{3}$ samples were tested, showing no sign of interaction in the spectrum.

Multi-layers fitting model for LSPRs. The distinct dielectric response of the core-shell NCs is implemented as an effective dielectric function $\varepsilon_{\text {eff }}(\omega)$ based on the Maxwell-Garnett effective medium approximation (EMA). This model is further extended to consider multiple shell regions and corresponding dielectric environments. We fit the experimental data with a particles warm optimization algorithm in MATLAB (R2020a. Natick, Massachusetts: The MathWorks Inc.) and we extract the carrier densities $n_{e, c o r e}$ and $n_{\text {e.shell }}$ and spatial extensions $\left(R_{\text {core }}, R_{\text {shell }}\right)$ of the core and shell regions, respectively, for each $\mathrm{NC}$ of increasing shell thickness before and after photodoping.

COMSOL simulations. Simulations for the energy band and carrier density profiles were solved numerically for spherical NCs using a finite-element method. Poisson's equation was solved with the software COMSOL Multiphysics v5.6 (Comsol Inc., Burlinghton MA USA) using a finite-element scheme (see Supplementary Information for details).

\section{Data availability}

All the data generated in this study has been deposited in the Zenodo database under accession code https://doi.org/10.5281/zenodo.5786683.

Received: 31 August 2021; Accepted: 23 December 2021; Published online: 27 January 2022

\section{References}

1. Yu, X., Marks, T. J. \& Facchetti, A. Metal oxides for optoelectronic applications. Nat. Mater. 15, 383-396 (2016)

2. Zandi, O. et al. Impacts of surface depletion on the plasmonic properties of doped semiconductor nanocrystals. Nat. Mater. 17, 710-717 (2017).

3. Gatti, T. et al. Opportunities from doping of non-critical metal oxides in last generation light-conversion devices. Adv. Energy Mater. 11, 2101041 (2021).

4. Ghini, M. et al. Photodoping of metal oxide nanocrystals for multi-charge accumulation and light-driven energy storage. Nanoscale 13, 8773-8783 (2021).

5. Lee, H. W. et al. Nanoscale conducting oxide PlasMOStor. Nano Lett. 14, 6463-6468 (2014).

6. Abb, M., Wang, Y., Papasimakis, N., de Groot, C. H. \& Muskens, O. L. Surface-enhanced infrared spectroscopy using metal oxide plasmonic antenna arrays. Nano Lett. 14, 346-352 (2014).

7. Johns, R. W. et al. Direct observation of narrow mid-infrared plasmon linewidths of single metal oxide nanocrystals. Nat. Commun. 7, 1-6 (2016).

8. Milliron, D. et al. Colloidal nanocrystal heterostructures with linear and branched topology. Nature 430, 190-195 (2004).

9. Gargas, D. J. et al. Engineering bright sub-10-nm upconverting nanocrystals for single-molecule imaging. Nat. Nanotechnol. 9, 300-305 (2014).

10. Llordés, A. et al. Linear topology in amorphous metal oxide electrochromic networks obtained via low-temperature solution processing. Nat. Mater. 15, 1267-1273 (2016).

11. Brozek, C. K. et al. Soluble supercapacitors: large and reversible charge storage in colloidal iron-doped ZnO nanocrystals. Nano Lett. 18, 3297-3302 (2018).

12. Tandon, B., Agrawal, A., Heo, S. \& Milliron, D. J. Competition between depletion effects and coupling in the plasmon modulation of doped metal oxide nanocrystals. Nano Lett. 19, 2012-2019 (2019).

13. Saleh, N. B. et al. Importance of doping, dopant distribution, and defects on electronic band structure alteration of metal oxide nanoparticles: Implications for reactive oxygen species. Sci. Total Environ. 568, 926-932 (2016).
14. Carroll, G. M., Schimpf, A. M., Tsui, E. Y. \& Gamelin, D. R. Redox potentials of colloidal n-type $\mathrm{ZnO}$ nanocrystals: effects of confinement, electron density, and Fermi-level pinning by aldehyde hydrogenation. J. Am. Chem. Soc. 137, 11163-11169 (2015).

15. Lounis, S. D., Runnerstrom, E. L., Bergerud, A., Nordlund, D. \& Milliron, D. J. Influence of dopant distribution on the plasmonic properties of indium tin oxide nanocrystals. J. Am. Chem. Soc. 136, 7110-7116 (2014).

16. Schimpf, A. M., Gunthardt, C. E., Rinehart, J. D., Mayer, J. M. \& Gamelin, D $\mathrm{R}$. Controlling carrier densities in photochemically reduced colloidal $\mathrm{ZnO}$ nanocrystals: Size dependence and role of the hole quencher. J. Am. Chem. Soc. 135, 16569-16577 (2013).

17. Gibbs, S. L., Staller, C. M. \& Milliron, D. J. Surface depletion layers in plasmonic metal oxide nanocrystals. Acc. Chem. Res. 52, 2516-2524 (2019).

18. Lounis, S. D., Runnerstrom, E. L., Llordés, A. \& Milliron, D. J. Defect chemistry and plasmon physics of colloidal metal oxide nanocrystals. J. Phys. Chem. Lett. 5, 1564-1574 (2014).

19. Staller, C. M. et al. Tuning nanocrystal surface depletion by controlling dopant distribution as a route toward enhanced film conductivity. Nano Lett. 18, 2870-2878 (2018).

20. Araujo, J. J. et al. Tunable band-edge potentials and charge storage in colloidal tin-doped indium oxide (ITO) nanocrystals. ACS Nano https://doi.org/10.1021/ acsnano.1c04660 (2021).

21. Schimpf, A. M., Lounis, S. D., Runnerstrom, E. L., Milliron, D. J. \& Gamelin, D. R. Redox chemistries and plasmon energies of photodoped In $2 \mathrm{O} 3$ and Sn-doped In2O3 (ITO) nanocrystals. J. Am. Chem. Soc. 137, 518-524 (2015).

22. Rinehart, J. D., Schimpf, A. M., Weaver, A. L., Cohn, A. W. \& Gamelin, D. R. Photochemical electronic doping of colloidal CdSe nanocrystals. J. Am. Chem. Soc. 135, 18782-18785 (2013).

23. Seiwatz, R. \& Green, M. Space charge calculations for semiconductors. J. Appl. Phys. 29, 1034-1040 (1958).

24. Sze, S. M. Physics of Semiconductor Devices. 2nd edn (Wiley, 1981).

25. Crockett, B. M., Jansons, A. W., Koskela, K. M., Johnson, D. W. \& Hutchison, J. E. Radial dopant placement for tuning plasmonic properties in metal oxide nanocrystals. ACS Nano 11, 7719-7728 (2017).

26. Wang, T. \& Radovanovic, P. V. Free electron concentration in colloidal indium tin oxide nanocrystals determined by their size and structure. J. Phys. Chem. C 115, 406-413 (2011)

27. Luther, J. M., Jain, P. K., Ewers, T. \& Alivisatos, A. P. Localized surface plasmon resonances arising from free carriers in doped quantum dots. Nat. Mater. 10, 361-366 (2011).

28. Agrawal, A. et al. Rationalizing the impact of surface depletion on electrochemical modulation of plasmon resonance absorption in metal oxide nanocrystals. ACS Photonics 5, 2044-2050 (2018).

29. Kanehara, M., Koike, H., Yoshinaga, T. \& Teranishi, T. Indium tin oxide nanoparticles with compositionally tunable surface plasmon resonance frequencies in the near-IR region. J. Am. Chem. Soc. 131, 17736-17737 (2009).

30. De Trizio, L. et al. Nb-doped colloidal TiO2 nanocrystals with tunable infrared absorption. Chem. Mater. 25, 3383-3390 (2013).

31. Gibbs, S. L. et al. Dual-mode infrared absorption by segregating dopants within plasmonic semiconductor nanocrystals. Nano Lett. 20, 7498-7505 (2020).

32. Kriegel, I. et al. Ultrafast photodoping and plasmon dynamics in fluorineindium codoped cadmium oxide nanocrystals for all-optical signal manipulation at optical communication wavelengths. J. Phys. Chem. Lett. 7, 3873-3881 (2016)

33. Haase, M., Weller, H. \& Henglein, A. Photochemistry and radiation chemistry of colloidal semiconductors. 23. Electron storage on $\mathrm{ZnO}$ patricles and size quantization. J. Phys. Chem. 92, 482-487 (1988).

34. Joost, U. et al. Reversible photodoping of $\mathrm{TiO} 2$ nanoparticles for photochromic applications. Chem. Mater. 30, 8968-8974 (2018).

35. Liao, L. et al. Theoretical study on the photodoping effects in la $1-x \mathrm{Sr} \times \mathrm{MnO}$ 3/SrNb y Ti 1-yO3 p-n heterojunction. Phys. Status Solidi Appl. Mater. Sci. 206, 1655-1659 (2009).

36. Zhang, H., Kulkarni, V., Prodan, E., Nordlander, P. \& Govorov, A. O. Theory of quantum plasmon resonances in doped semiconductor nanocrystals. J. Phys. Chem. C 118, 16035-16042 (2014).

37. Ghini, M., Camellini, A., Rubino, A. \& Kriegel, I. Multi-charge transfer from photodoped ITO nanocrystals. Nanoscale Adv. https://doi.org/10.1039/ D1NA00656H (2021).

38. Jansons, A. W. \& Hutchison, J. E. Continuous growth of metal oxide nanocrystals: enhanced control of nanocrystal size and radial dopant distribution. ACS Nano 10, 6942-6951 (2016).

39. Rueden, C. T. et al. ImageJ2: ImageJ for the next generation of scientific image data. BMC Bioinformatics 18, 529 (2017).

40. Fairley, N. et al. Systematic and collaborative approach to problem solving using X-ray photoelectron spectroscopy. Appl. Surf. Sci. Adv. 5, 100112 (2021). 


\section{Acknowledgements}

For this work, the authors acknowledge the support of both European Union's Horizon 2020 European Research Council, under grant agreement no. 850875 (I.K.) (LightDYNAMO), and European Union's Horizon 2020 Research and Innovation program under grant agreement no. 101017821 (I.K.) (LIGHT-CAP). The authors thank Dr Ankit Agrawal for useful discussion, the Electron Microscopy Facility of the Italian Institute of Technology for support with material characterization, and F. Drago (Nanochemistry department) for the ICP measurements.

\section{Author contributions}

M.G. and N.C. contributed equally to this work. M.G. designed the experiment, synthesized and characterized the nanomaterial, performed photodoping and titration experiments, developed the multi-layer optical model, and wrote the manuscript. N.C. coordinated the research work, performed numerical simulations, analyzed the experimental data and wrote the manuscript. M.B.L. performed numerical simulations; N.P. developed the multi-layer optical model. M.W. performed titration experiments; M.P. performed XPS measurements and analyzed the data. A.F. and L.M. supervised the research. I.K. designed the experiment, supervised and coordinated all research work and wrote the manuscript.

\section{Competing interests}

The authors declare no competing interests.

\section{Additional information}

Supplementary information The online version contains supplementary material available at https://doi.org/10.1038/s41467-022-28140-y.
Correspondence and requests for materials should be addressed to Nicola Curreli or Ilka Kriegel.

Peer review information Nature Communications thanks the anonymous reviewers for their contribution to the peer review of this work.

Reprints and permission information is available at http://www.nature.com/reprints

Publisher's note Springer Nature remains neutral with regard to jurisdictional claims in published maps and institutional affiliations.

(c) Open Access This article is licensed under a Creative Commons Attribution 4.0 International License, which permits use, sharing, adaptation, distribution and reproduction in any medium or format, as long as you give appropriate credit to the original author(s) and the source, provide a link to the Creative Commons license, and indicate if changes were made. The images or other third party material in this article are included in the article's Creative Commons license, unless indicated otherwise in a credit line to the material. If material is not included in the article's Creative Commons license and your intended use is not permitted by statutory regulation or exceeds the permitted use, you will need to obtain permission directly from the copyright holder. To view a copy of this license, visit http://creativecommons.org/ licenses/by/4.0/.

(C) The Author(s) 2022 